\title{
Impaired phosphorylation and mis-localization of Bub1 and BubR1 are responsible for the defective mitotic checkpoint function in Brca2-mutant thymic lymphomas
}

Hyunsook Lee

Division of Molecular Life Sciences

Ewha Womans University

11-1 Daehyun-dong, Seodaemoon-gu

Seoul 120-750, Korea

Tel, 82-2-3277-4335; Fax, 82-2-3277-3760;

E-mail, hyunlee@ewha.ac.kr

Accepted 1 September 2003

Abbreviations: BRCA2, human BRCA2; Brca2, murine BRCA2

\begin{abstract}
Breast cancer susceptibility gene, BRCA2, is a tumor suppressor and individuals who inherit one defected copy of BRCA2 allele experience early onset breast cancer or ovarian cancer accompanied by the loss of the wild type allele. Mouse model for Brca2 mutation shows growth retardation and paradoxical occurrence of thymic lymphomas. Thymic lymphomas from Brca2-mutant mice harbor mutations in p53, Bub1, and $B u b R 1$, which function as mitotic checkpoint proteins. Therefore, interplay between Brca2 and mitotic checkpoint has been suggested in the maintenance of genetic fidelity, although it has not been assessed whether the unique mutations in Bub1 and BubR1 found in Brca2-mutant mice are responsible for the abolishment of mitotic checkpoint function. This report demonstrates that Bub1 and BubR1 mutant proteins from Brca2 ${ }^{-1}$ thymic lymphomas have defects in the phosphorylation and kinetochore localization after spindle damage. Thus, the mutations of Bub1 and BubR1 found in Brca2mutant mice indeed are responsible for the chromosome instability in Brca2-mutated tumors.
\end{abstract}

Keywords: breast neoplasms; BRCA2 protein; mitosis; lymphoma; tumor suppressor proteins; T-cell; tumor

\section{Introduction}

Individuals who inherit one defective copy of BRCA2 gene are predisposed to early onset breast cancer and ovarian cancer (Wooster et al., 1995; Tavtigian et al., 1996). Tumor cells from predisposed individuals usually contain mutations in both alleles (Collins et al., 1995; Gudmundsson et al., 1995), thus BRCA2 works as a tumor suppressor. The function of BRCA2 has been largely ascribed to its role in DNA repair. BRCA2 binds to Rad51, a RecA homologue involved in homologous recombination and double-strand break repair (Venkitaraman, 2001; 2002). Murine cells homozygous for Brca2 truncations in exon 10 (Ludwig et al., 1997; Sharan et al., 1997; Suzuki et al., 1997) or exon 11 (Connor et al., 1997; Friedman et al., 1998) exhibit hypersensitivity to $\gamma$-irradiation, although checkpoint response to DNA damage is largely intact (Patel et al., 1998).

Mice homozygous for Brca2 truncations exhibit growth retardation due to activation of checkpoint response (Patel et al., 1998). Paradoxically, they develop thymic lymphomas (Connor et al., 1997; Friedman et al., 1998), and it has been shown previously, that the tumorigenesis in $\mathrm{Brca} 2^{\mathrm{Tr} / \mathrm{Tr}}$ mice involves inactivation of mitotic checkpoint function and exhibit chromosomal instability (Lee et al., 1999). Thus, it is likely that inactivating mutations in mitotic checkpoint genes cooperate with BRCA2 deficiency in the pathogenesis of inherited breast cancer.

Mitotic checkpoint, also called spindle assembly checkpoint, delays the onset of anaphase until all chromosomes are attached with spindle, and ensures proper chromosome segregation to the daughter cells (Rudner and Murray, 1996; Taylor, 1999). Mitotic checkpoint is activated by the presence of unattached kinetochores with spindle (Rudner and Murray, 1996). Lack of microtubule attachment or lack of tension at the kinetochore activates phosphorylation of kinases that activate components of mitotic checkpoint, Bub1, Bub3, Mad 1-3, and Mps1 (Rudner and Murray, 1996), at the kinetochore. Chromosome loss or gain due to defects in mitotic checkpoint can kill cells, cause birth defects or contribute to tumorigenesis.

Progression to cancer required gross changes in genetic information. Calculations using human nongermline cells show the mutation rate of $10^{-7}$ per gene per cell generation (Loeb, 1991). However, the appearance of cancer cells required mutations of half-dozen or more mutations in cellular genes, which directly or indirectly affect proliferation. Hence, with the normal mutation rate, tumor formation is impos- 
sible during a human life time. Dysfunctional BRCA2 enforces mutagenic repair (Venkitaraman, 2001) that activates checkpoint response and apoptosis. Surviving cells that acquired mutations in mitotic checkpoint function are exposed to another level of change in genetic information, aneuplody, resulting from uncontrolled chromosome segregation. Therefore, cooperation of truncation in Brca2 and mutation in mitotic checkpoint results in gross change in genetic information that in effect achieves high mutability and progress to early onset breast and ovarian cancer.

Previous report showed that the tumors from Brca2deficient mouse had defects in mitotic checkpoint and concurrently found mutations in p53, Bub1, and BubR1. However, whether the unique Bub1 and BubR1 mutations found in Brca2-deficient mouse contribute to neoplastic transformation has not been formally assessed in the previous report. In this report, a Brca2deficeint $T$ cell lymphoma showing defects in the phosphorylation of mitotic checkpoint components Bub1 and BubR1 is described. Furthermore, another Brca2deficeint thymic lymphoma showed mis-localization of Bub1 in response to microtubule disruption, demonstrating that mitotic checkpoint is indeed not functioning in Brca2-mutant tumors. These results strongly support the notion that mitotic checkpoint inactivation accelerates the chromosome instability in BRCA2mutant cells.

\section{Materials and Methods}

\section{Culture of thymic lymphomas in $\mathrm{Brca} 2^{\mathrm{Tr} / \mathrm{Tr}}$ mice}

The Brca $2^{\text {Tr/Tr}}$ homozygous mice that survived weaning developed thymic lymphomas between 12 to 14 weeks of age. Single cell suspensions were made from each thymus by passing through the syringe without a needle. Cells were cultured with RPMI medium containing $50 \mathrm{nM}$ of $\beta$-mercaptoethanol, $10 \%$ FBS, IL-2 (Roche Biochemicals, Germany) according to the recommended concentration. The next day, cells were washed by centrifugation in $1,000 \mathrm{rpm}$ for $5 \mathrm{~min}$. The pellet was once again resuspended in RPMI medium described above. After several weeks of culture in the cytokine-containing medium, cells were tested of their viability in culture in the presence or absence of cytokines by the MTT assay (Roche Biochemicals).

\section{Western blot analysis of Bub1 and BubR1}

$2 \times 10^{4}$ cells were either treated or untreated with 200 $\mathrm{ng} / \mathrm{ml}$ nocodazole for $24 \mathrm{~h}$ and subjected to Western blotting. Human monoclonal antibody to BubR1 or Bub1 were kind gifts of Dr. Frank MaKeon and Dr. Stephen Taylor (Harvard Medical School). For loading control, the blot was reprobed with anti- $\beta$-actin $(A b-2$,
Calbiochem).

\section{Immunohistochemistry}

Suspension cell cultures were treated with $250 \mathrm{ng} / \mathrm{ml}$ of nocodazole for $24 \mathrm{~h}$ before fixation. At the indicated time point, suspension cells were put onto the poly-L-lysine (Sigma) coated cover slips. Nocodazole treated cells were rinsed twice in room temperature with phem $(60 \mathrm{mM}$ Pipes, $25 \mathrm{mM}$ Hepes, $\mathrm{pH} 7.0,10$ $\mathrm{mM}$ EGTA, $4 \mathrm{mM} \mathrm{MgSO}_{4}$ ) (phem). Cells were extracted for $5 \mathrm{~min}$ in $0.5 \%$ triton X-100 (Sigma) in phem. Then cells were fixed with $1 \%$ paraformaldehyde, freshly prepared in phem for 15 minutes followed by rinsing twice in mbst $(10 \mathrm{mM}$ Mops, $\mathrm{pH} 7.3,150 \mathrm{mM}$ $\mathrm{NaCl}, 0.05 \%$ Tween-20 (mbst)). Blocking was processed in $20 \%$ boiled goat serum in mbst for $1 \mathrm{~h}$ followed by 1:100 dilution of Bub1 antibody incubation. After antibody staining, cells were counter-stained with propidium iodide for visualization of DNA. Localization of Bub1 was visualized by the observation under the confocal microscopy using Bio-Rad MRC 1,000.

\section{Results and Discussion}

Previously, using Brca2 ${ }^{\text {Tr/Tr }}$ mice (Friedman et al., 1998), we have shown that mitotic checkpoint inactivation overcomes growth retardation in Brca2 ${ }^{\mathrm{Tr} / \mathrm{Tr}} \mathrm{MEFs}$ and fosters transformation. Furthermore, mutations in $p 53$, Bub1, or BubR1 are found in Brca $2^{\operatorname{Tr} / T r}$ thymic lymphomas, indicating that mitotic checkpoint function is lost (Lee et al., 1999). However, it has not been assessed in which way mutations in Bub1 and BubR1 affect mitotic checkpoint response, and whether they are dominant negative mutants.

To address this issue, analysis on the status and function of mitotic checkpoint proteins Bub1 and BubR1 were performed in Brca2 $2^{\text {Tr/Tr }}$ thymic lymphomas. First, cells isolated from three independent Brca $2^{\text {Tr/Tr }}$ thymus were cultured for 5 days and analyzed for their surface markers. The cells display markers characteristic of an early stage of thymic T cell development (Figure 1). They are positive for TCR $\beta$ and Thy1, indicating that they are T-cell originated. In addition, when these cells were stained with CD4 and CD8 antibodies, they showed negative staining for CD4, but stained weakly with CD8 (CD4 $4^{-}$CD ${ }^{\text {low; }}$; lymphoma A) (Figure 1). Second and third thymic lymphomas isolated from different Brca2 $2^{T r / T r}$ mice revealed that one was CD4 single positive (CD4 ${ }^{+}$CD8; lymphoma $B$ ), and the other was CD8 single positive (CD4- CD8 ${ }^{+}$; lymphoma C) (data not shown). These data suggest that Brca2 disruption in mice did not interfered with early $T$ cell differentiation since the thymic lymphomas were monoclonal and showed dif- 

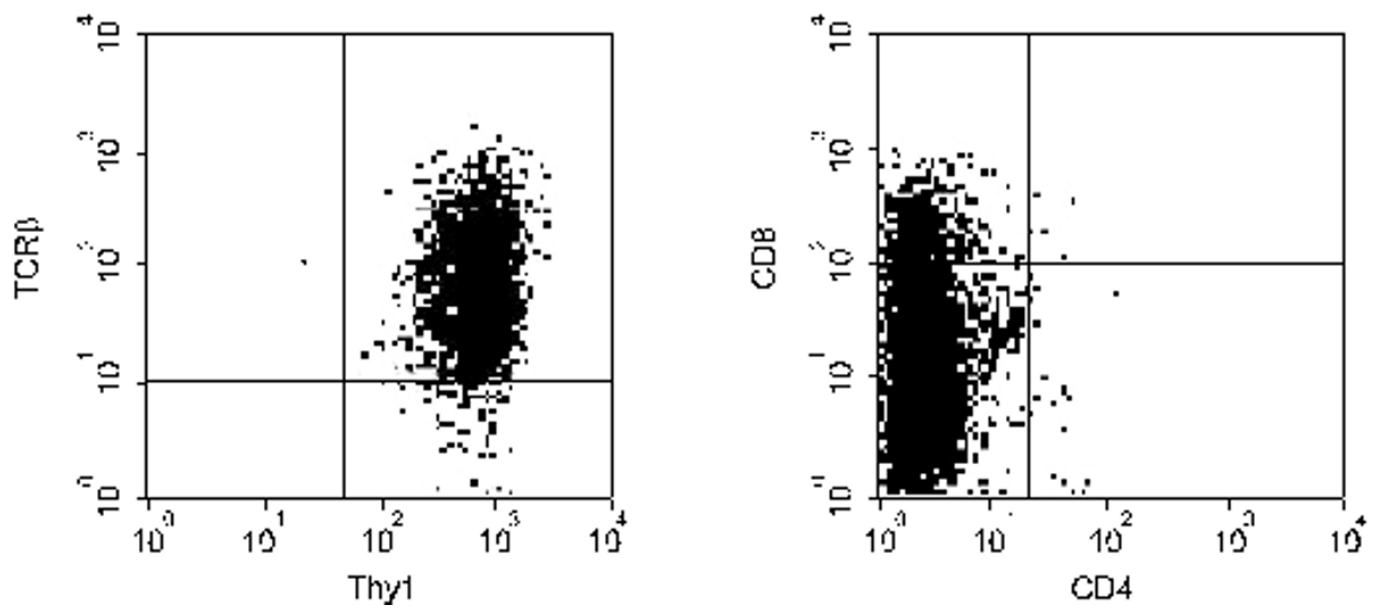

Figure 1. Cells isolated from Brca2 ${ }^{1 / I r}$ thymus show markers characteristic of early $\mathrm{T}$ cell differentiation.

ferent stages of $T$ cell differentiation. Unlike normal thymocytes, they divide continuously in culture independent of cytokine growth factors (data not shown).

As discussed in the previous report (Lee et al., 1999), all three of Brca2 $2^{\text {Tr/Tr }}$ thymic lymphomas used in this study, exhibit defects in mitotic checkpoint function after spindle disruption. Karyotyping of these thymic lymphomas revealed that Brca2 ${ }^{\mathrm{Tr} / \mathrm{Tr}}$ lymphoma cells exhibit aberrant chromosome number and structure, characterized as chromosome instability (Lee et al., 1999). Interestingly, sequencing result revealed that one mutated Bub1 allele and wild-type BubR1 are found in lymphoma $A$ and lymphoma B. Lymphoma $C$ has wild type Bub1 and one mutated allele of BubR1 (Lee et al., 1999). In order to test if Bub1 and $B u b R 1$ mutations in Brca2 ${ }^{T r / T r}$ thymic lymphomas are responsible for the inactivation of mitotic checkpoint function, phosphorylation status was investigated after nocodazole treatment. Bub1 kinase is autophosphorylated upon spindle disruption, or lack of tension at the kinetochore, in yeast (Roberts et al., 1994; Farr and Hoyt, 1998), and the autophoshprylation is required for its kinase activity (Roberts et al., 1994; Taylor et al., 1998). Therefore, the phosphorylation status of Bub1 in Brca2 $2^{\mathrm{Tr} / \mathrm{Tr}}$ thymic lymphomas was tested to ask if it is functional. Upon nocodazole treatment, in control Pim-1 T cell lymphomas (Jonkers et al., 1997), up-shifted phosphorylated Bub1 band was detected by Western blotting with a monoclonal antibody to murine Bub1 (Taylor and McKeon, 1997) (Figure 2). Phosphatase treatment revealed that the shifted band is indeed a product of phosphorylation (data not shown). In lymphoma $A$ and $C$, phosphorylated band was also detected upon nocodazole treatment, suggesting that Bub1 phosphorylation after spindle disruption is intact in these cells (Figure 2). However, in lymphoma $B$, the level of Bub1 was

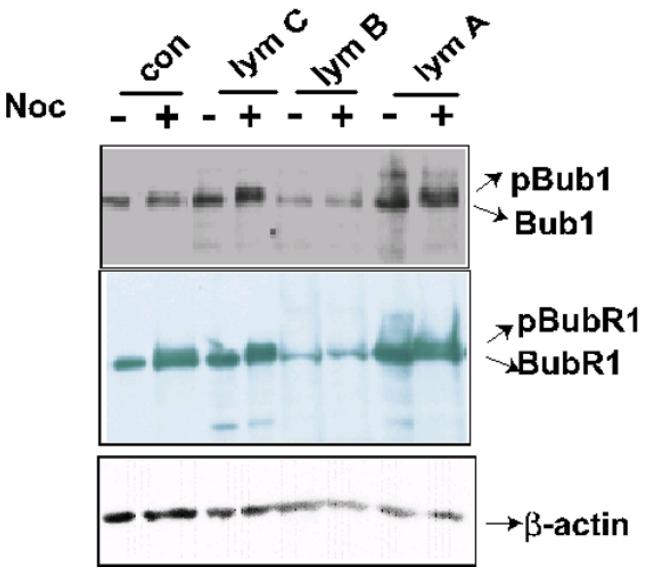

Figure 2. Mitotic checkpoint proteins Bub1 and BubR1 in Brca2 ${ }^{1 / / 1 /}$ cells show altered response to microtubule disruption. Bub1 and BubR1 in control cells (lymphomas from Pim-1 transgenic), lymphoma $C$, and lymphoma $A$ show phosphorylated forms (shifted bands) upon nocodazole treatment. The level of $\beta$-actin in the lower panel indicates that the protein amounts loaded on each lane are approximately equal.

significantly low and phosphorylation was lost upon nocodazole treatment. Reprobing the blot with antibody to $\beta$-actin revealed that similar amounts of protein were loaded (Figure 2). Therefore, it is likely that the machinery that controls Bub1 level and phosphorylation is altered in lymphoma B. Since lymphoma $A$ and $B$ both have one mutated allele of Bub1, and yet Bub1 phosphorylation in lymphoma $A$ is intact, it is speculated that an unknown mechanism in lymphoma $B$ is deregulated that in effect resulted in a low level of Bub1 and inability of phosphorylation (Figure 2).

BubR1 has been cloned by its ability to bind to Bub3 (Taylor et al., 1998). Simultaneously, its muta- 
tion has been found in certain colon cancer cell lines that exhibited chromosome instability (Cahill et al., 1998). Of note, mutation in BubR1 is also found in Brca2 ${ }^{\mathrm{Tr} / \mathrm{Tr}}$ lymphomas. To ask if similar defect in phophorylation is present in $\mathrm{Brca} 2^{\mathrm{Tr} / \mathrm{Tr}}$ lymphomas, the blot used to detect Bub1 was reprobed with antibody to BubR1. Since the antibody to BubR1 does not cross-react with Bub1 (personal communications with Dr. S. Taylor), the blot shown in Figure 2 reveals the level of murine BubR1. Comparing with control Pim-1 lymphoma (Jonkers et al., 1997), phosphorylation of BubR1 upon spindle disruption is more or less intact in lymphoma C (Figure 2, middle panel). High level of BubR1 was detected in lymphoma $A$, and the phosphorylation of BubR1 upon nocodazole treatment was intact. Interestingly, BubR1 level was $10^{\text {th }}$ the level in lymphoma $B$ and the phosphorylation after microtubule disruption was absent, as Bub1 was (Figure 2). Note that sequencing result showed that BubR1 in lymphoma B was wild type (Lee et al., 1999). Taken together, it is very likely that an unidentified mechanism which regulates Bub1 level and phosphorylation is impaired in lymphoma B. And as a result, BubR1 level and phosphorylation is impaired, since BubR1 is likely to act downstream of Bub1 (Rudner and Murray, 1996; Taylor, 1999; Taylor et al., 1998). It is speculative that at least in lymphoma $B$, defect in phosphorylation of Bub1 and BubR1 that governs the activation of mitotic checkpoint kinases, is responsible for inactivation of mitotic checkpoint function in Brca2 ${ }^{\operatorname{Tr} / \mathrm{Tr}}$ thymic lymphoma.

Mitotic checkpoint proteins function at the kinetochore since they sense and check if all the chromosomes are aligned at the metaphase plane and attached with spindles, before the onset of anaphase (Rudner and Murray, 1996). One way to detect defective mitotic checkpoint function is to test whether the protein correctly localizes to the kinetochores when microtubule spindles are disrupted. It has been shown that $3 \mathrm{~F} 3 / 2$ staining is absent in $\mathrm{Brca} 2^{\mathrm{Tr} / \mathrm{Tr}}$ lymphomas after spindle disruption (Lee et al., 1999). 3F3/2 anti-phosphoepitope antibody recognizes kinetochores that are not attached to the spindle (Campbell and Gorbsky, 1995; Nicklas et al., 1995; Daum et al., 2000). Thus, lack of $3 F 3 / 2$ staining at the kinetochore after nocodazole treatment supports the notion that mitotic checkpoint is not functioning in Brca $2^{\mathrm{Tr} / \mathrm{Tr}}$ lymphomas. However, 3F3/2 staining does not directly speak to the functionality of Bub1 and BubR1, since the epitope detected by $3 F 3 / 2$ is not Bub1 nor BubR1 (Daum and Gorbsky, 1998; Daum et al., 2000).

To directly test if mutant Bub1 in $\mathrm{Brca}^{\mathrm{Tr} / \mathrm{Tr}} \mathrm{lym}$ phoma cells were functional, lymphoma A were treated with nocodazole and stained with anti-Bub1. As seen in Figure 3, Bub1 colocalized with propidium iodide stained DNA when treated with nocodazole in

\section{Ctrl Brca2 Tr/Tr Liphoma Líphoma}

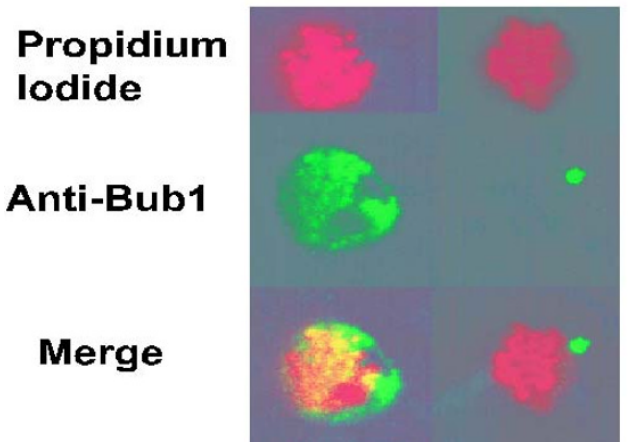

Figure 3. Mis-localization of Bub1 in Brca2 ${ }^{\text {Ir/Ir }}$ lymphoma A after nocodazole treatment. Nocodazole treatment in control cells causes costaining of Bub1 (green in color) with DNA stained by propidium iodide (colored in red). In contrast, in Brca2Tr/Tr lymphoma A, Bub1 fails to localize to the kinetochore.

control Pim-1 transgenic lymphoma cells. In contrast, propidium iodide and Bub1 staining is not merged into one picture in Brca2 ${ }^{\mathrm{Tr} / \mathrm{Tr}}$ thymic lymphoma. This result shows that the genetic mutation in Bub1, found in Brca2 ${ }^{\mathrm{Tr} / \mathrm{Tr}}$ thymic lymphoma $A$, encodes a dominant negative mutant protein, since one mutated allele is sufficient for mis-localization of Bub1.

The data presented here show that mitotic checkpoint kinase Bub1 and BubR1 are functioning as dominant negative mutants in Brca $2^{\mathrm{Tr} / \mathrm{Tr}}$ lymphomas, either by defect in phosphorylation or localization to the kinetochore. In lymphoma $\mathrm{C}$, heterozygous mutation in BubR1 is found (Lee et al., 1999). In Western blot, a molecule of $90 \mathrm{kDa}$, in addition to the wild type $119 \mathrm{kDa}$, is detected unlike controls. Although the consequences of the mutations need to be investigated further, mitotic checkpoint function assayed by $3 F 3 / 2$ staining showed absence of phosphorylation at the kinetochore in lymphoma C (Lee et al., 1999). In summary, all three thymic lymphomas showed defect in mitotic checkpoint function after spindle damage. However, attempts to detect Bub1 staining in lymphoma B was not successful, probably due to low level of Bub1. Therefore, whether phosphorylation of Bub1 is required for proper kinetochore localization or vice versa remains to be investigated further. The mechanism underlying the difference between lymphoma $A$ and $B$ with same Bub1 mutation is not yet solved. Since phosphorylation of Bub1 in lymphoma A was intact yet showed mis-localization of Bub1, it is possible that phosphorylation and localization of Bub1 is a separate event. It is also possible that in addition to Bub1 and BubR1 mutations, other unknown regulatory molecules have been mutated that resulted in the difference between lymphoma $A$ and 
lymphoma $B$.

Most tumor cells exhibit aneuploidy early or late in their progression to cancer. In this regard, it is important to note that accumulating reports suggest the involvement of mitotic checkpoint dysfunction in tumorigenesis. Certain types of cancer that show aneuploidy earlier in their tumorigenesis step are thought to involve mutations in checkpoints that monitor chromosome segregation (Cahill et al., 1998; Lee et al., 1999; Ru et al., 2002).

The data presented in this report support the notion that mutations in mitotic checkpoint components contribute to genetic instability by deregulated chromosome segregation, which results in gross genetic information change by chromosome gain or loss. Bub1 and BubR1 mutation resulted in defected phosphorylation and mis-localization. The consequences of these events would result in massive alteration in chromosome number that normal cells cannot endure. It should be stressed that the mutations in Bub1 and BubR1 have been found in Brca2-deficient background. In Brca2 mutated cells, mutation rate can increase, that in a subset of cells critical mutations in mitotic checkpoint can take place that overcome growth retardation and apoptosis. Thus, this report suggest that Brca2-involved repair machinery and Bub1/ BubR1-contained mitotic checkpoint machinery crosstalk in keeping the genetic integrity and cell survival.

\section{Acknowledgement}

I am grateful to Prof. Ashok Venkitaraman (Cambridge University, UK) for discussions and advice throughout the work. I am also grateful to Dr. Frank McKeon (Harvard Medical School, Boston) and Dr. Stephen Taylor (Univ. of Manchester, UK) for providing Bub1 and BubR1 monoclonal antibodies. This work was partly supported by funding from the National Cancer control program by the Korean ministry of Health and Wellfare.

\section{References}

Cahill DP, Lengauer C, Yu J, Riggins GJ, Willson JK, Markowitz SD, Kinzler KW, Vogelstein B. Mutations of mitotic checkpoint genes in human cancers. Nature 1998;392:300-3

Campbell MS, Gorbsky GJ. Microinjection of mitotic cells with the 3F3/2 anti-phosphoepitope antibody delays the onset of anaphase. J Cell Biol 1995;129:1195-204

Collins N, McManus R, Wooster R, Mangion J, Seal S, Lakhani SR, Ormiston W, Daly PA, Ford D, Easton DF, et al. Consistent loss of the wild type allele in breast cancers from a family linked to the BRCA2 gene on chromosome 13q12-13. Oncogene 1995;10:1673-5

Connor F, Bertwistle D, Mee PJ, Ross GM, Swift S, Grigorieva E, Tybulewicz VL, Ashworth A. Tumorigenesis and a DNA repair defect in mice with a truncating Brca2 mutation. Nat Genet 1997;17:423-30

Daum JR, Gorbsky GJ. Casein kinase II catalyzes a mitotic phosphorylation on threonine 1,342 of human DNA topoisomerase II alpha, which is recognized by the $3 F 3 / 2$ phosphoepitope antibody. J Biol Chem 1998;273:30622-9

Daum JR, Tugendreich S, Topper LM, Jorgensen PM, Hoog C, Hieter P, Gorbsky GJ. The 3F3/2 anti-phosphoepitope antibody binds the mitotically phosphorylated anaphase-promoting complex/cyclosome. Curr Biol 2000;10:R850-2

Farr KA, Hoyt MA. Bub1p kinase activates the Saccharomyces cerevisiae spindle assembly checkpoint. Mol Cell Biol 1998p;18:2738-47

Friedman LS, Thistlethwaite FC, Patel KJ, Yu VP, Lee H, Venkitaraman AR, Abel KJ, Carlton MB, Hunter SM, Colledge $\mathrm{WH}$, et al. Thymic lymphomas in mice with a truncating mutation in Brca2. Cancer Res 1998;58:1338-43

Gudmundsson J, Johannesdottir G, Bergthorsson JT, Arason A, Ingvarsson S, Egilsson V, Barkardottir RB. Different tumor types from BRCA2 carriers show wild-type chromosome deletions on 13q12-q13. Cancer Res 1995;55:4830-2

Jonkers J, Korswagen HC, Acton D, Breuer M, Berns A. Activation of a novel proto-oncogene, Frat1, contributes to progression of mouse T-cell lymphomas. Embo J 1997;16: $441-50$

Lee $\mathrm{H}$, Trainer AH, Friedman LS, Thistlethwaite FC, Evans MJ, Ponder BA, Venkitaraman AR. Mitotic checkpoint inactivation fosters transformation in cells lacking the breast cancer susceptibility gene, Brca2. Mol Cell 1999;4:1-10

Loeb LA. Mutator phenotype may be required for multistage carcinogenesis. Cancer Res 1991;51:3075-9

Ludwig T, Chapman DL, Papaioannou VE, Efstratiadis A. Targeted mutations of breast cancer susceptibility gene homologs in mice: lethal phenotypes of Brca1, Brca2, Brca1/ Brca2, Brca1/p53, and Brca2/p53 nullizygous embryos. Genes Dev 1997;11:1226-41

Nicklas RB, Ward SC, Gorbsky GJ. Kinetochore chemistry is sensitive to tension and may link mitotic forces to a cell cycle checkpoint. J Cell Biol 1995;130:929-39

Patel KJ, Yu VP, Lee H, Corcoran A, Thistlethwaite FC, Evans MJ, Colledge WH, Friedman LS, Ponder BA, Venkitaraman AR. Involvement of Brca2 in DNA repair. Mol Cell 1998;1:347-57

Roberts BT, Farr KA, Hoyt MA. The Saccharomyces cerevisiae checkpoint gene BUB1 encodes a novel protein kinase. Mol Cell Biol 1994;14:8282-91

Ru HY, Chen RL, Lu WC, Chen JH. hBUB1 defects in leukemia and lymphoma cells. Oncogene 2002;21:4673-9

Rudner AD, Murray AW. The spindle assembly checkpoint. Curr Opin Cell Biol 1996;8:773-80

Sharan SK, Morimatsu M, Albrecht U, Lim DS, Regel E, Dinh C, Sands A, Eichele G, Hasty P, Bradley A. Embryonic lethality and radiation hypersensitivity mediated by Rad51 in mice lacking Brca2. Nature 1997;386:804-10

Suzuki A, de la Pompa JL, Hakem R, Elia A, Yoshida R, 
Mo R, Nishina H, Chuang T, Wakeham A, Itie A, et al. Brca2 is required for embryonic cellular proliferation in the mouse. Genes Dev 1997;11:1242-52

Tavtigian SV, Simard J, Rommens J, Couch F, ShattuckEidens D, Neuhausen S, Merajver S, Thorlacius S, Offit K, Stoppa-Lyonnet D, et al. The complete BRCA2 gene and mutations in chromosome 13q-linked kindreds. Nat Genet 1996;12:333-7

Taylor SS. Chromosome segregation: dual control ensures fidelity. Curr Biol 1999;9:R562-4

Taylor SS, Ha E, McKeon F. The human homologue of Bub3 is required for kinetochore localization of Bub1 and a Mad3/ Bub1-related protein kinase. J Cell Biol 1998;142:1-11
Taylor SS, McKeon F. Kinetochore localization of murine Bub1 is required for normal mitotic timing and checkpoint response to spindle damage. Cell 1997;89:727-35

Venkitaraman AR. Chromosome stability, DNA recombination and the BRCA2 tumour suppressor. Curr Opin Cell Biol 2001;13:338-43

Venkitaraman AR. Cancer susceptibility and the functions of BRCA1 and BRCA2. Cell 2002;108:171-82

Wooster R, Bignell G, Lancaster J, Swift S, Seal S, Mangion J, Collins N, Gregory S, Gumbs C, Micklem G. Identification of the breast cancer susceptibility gene BRCA2. Nature 1995;378:789-92 